Products and Services In Practice is provided to readers using text and images from the manufacturer, supplier or distributor and does not imply endorsement by $B D J$ In Practice. Normal and prudent research should be exercised before purchase or use of any product mentioned.

Please send product and services news through to David Westgarth, BDJ In Practice via: David.Westgarth@bda.org

\section{Set yourself apart}

Looking to differentiate and take your career to the next level?

Ucer Education's Postgraduate Certificate (PGCert) in Implant Dentistry (EduQual Level 7) celebrates a quarter century of excellence this year, having built a rocksolid reputation under Specialist Oral Surgeon, Professor Cemal Ucer.

If you're interested in developing your knowledge and skills in the field, you'll receive top-notch guidance under a great mentor with the PGCert in Implant Dentistry. There will be both practical, high-value hands-on training and theoretical teaching to ensure you build your confidence and skills. Set yourself apart with clinical training and support you need to truly thrive, with Ucer Education.

For more information on the PG Cert in Implant Dentistry from Ucer Education - supported by Geistlich, Megagen, Neoss, TRI Implants and General Medical please visit www.ucer.education or call Prof Ucer on 07767645331.

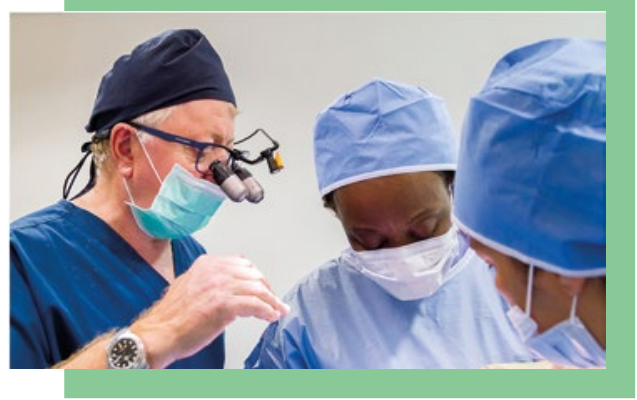

\title{
Annual forum details announced
}

Align Technology, Inc. (NASDAQ: ALGN) has announced that it will stage its annual UK GP Forum on 6 February 2021.

The event will be broadcast online from a TV studio. The company has curated a line-up of speakers from both inside and outside of dentistry to explore the most pressing topics shaping dental sector, which has evolved throughout 2020. The event's programme has been designed to encourage dentists to explore insights into the latest technology and product innovations from Align Technology and beyond.

The Invisalign GP Forum will showcase relatable strategies for dental professionals on how to break down barriers to adoption, highlighting the clinical, business and patient centric benefits of digital-centric practice. The topics have been chosen to inspire forward thinking dentists to become dynamic players for 2021 and beyond.

To book a place on the Invisalign UK GP Forum, visit: www.ukgpforum.co.uk

\section{The game-changing COVID-19 antibody test}

Screening your dental team for COVID-19 is quick and effective with the BioSURECOVID19 Triple Antibody Rapid Test. It is simple to perform and only requires $5 \mathrm{ul}$ of capillary blood to deliver results in just 10 minutes.

Developed and manufactured in the UK, this test comes in a box - priced at $£ 750$ excluding VAT - that contains sufficient

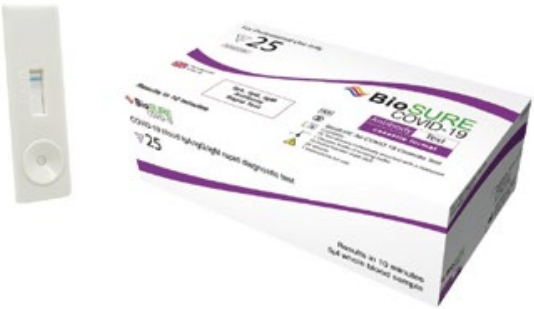

product for 25 tests ( $£ 30$ per test). Take full advantage of this game-changing solution with the help of leading oral health specialist, Curaden. The team can support you with:

$\rightarrow$ High-quality PPE

$\rightarrow$ Educational resources

$\rightarrow$ Comprehensive training for your COVID-19 screening staff

$\rightarrow$ Creating a socially distanced screening area in your practice

$\rightarrow$ Reporting tools in the form of the BioSURE powered by PocDoc app.

To order, please call 01480862084 , email info@curaprox.co.uk or visit www.curaprox.co.uk.

\section{On trend cleaning}

With health and hygiene is at the forefront of everyone's mind your patients are looking for safe, reliable products and brands they can trust. Arm \& Hammer has a fine pedigree for manufacturing high quality products with the clinically proven cleaning powers of baking soda.
By recommending the range of Arm \& Hammer baking soda toothpastes, you can offer your patients the benefits of a gentle but effective deep clean that is designed to address their specific dental needs and improve their oral health. For more information about the carefully formulated
Arm \& Hammer toothpaste range, please visit http://www.armandhammer.co.uk/ or email ukenquiries@churchdwight.com.

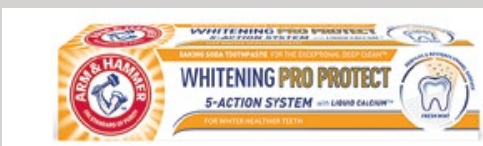

